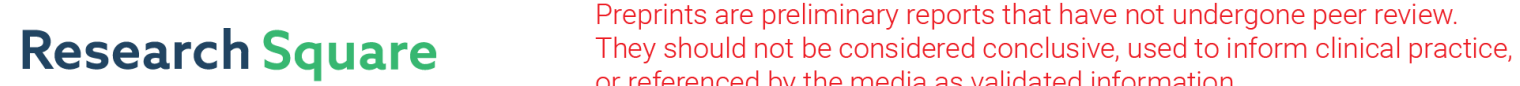 or referenced by the media as validated information. \\ The Lifting Force of An Airplane Wing When Flying Horizontally at High Speeds.
}

Alexander Braginsky ( $\square$ a.braginsky@mail.ru )

SFU, Institute of Physics

\section{Research}

Keywords: lifting force, airfoil, vortex trail

Posted Date: August 7th, 2020

DOI: https://doi.org/10.21203/rs.3.rs-51613/v1

License: (c) (i) This work is licensed under a Creative Commons Attribution 4.0 International License. Read Full License 


\title{
The lifting force of an airplane wing when flying horizontally at high speeds.
}

\author{
Alexander Braginsky \\ SFU, Institute of Physics, 344090, Rostov-on-Don, Stachki 194 \\ e-mail: a.braginsky@mail.ru
}

\begin{abstract}
In this paper, an explanation is given of the lift force of an airplane during horizontal flight. It is shown that during a flight, five vertical forces act on the airplane: gravity; pressure gradient with a minus sign; Archimedes force; potential force and the vortex force obtained from the action minimum. The first three forces were known before. The potential force was also known from the Bernoulli equation, but its effect on the airfoil from the air had not previously been taken into account. The vortex force obtained from the minimum action in the application to a continuous medium was not taken into account in aerodynamics. In horizontal flight the vortex force is directed upwards, it compensates for the gravity of the airplane at high speed commensurate with the speed of sound. The article provides an explanation of the vortex trail behind the airplane, mentioned in the CMI Millennium problem.
\end{abstract}

Keywords: lifting force; airfoil; vortex trail.

\section{Introduction}

The idea to write this paper came after Scientific American published an article: "No one can explain why planes stay in the air" [1]. In [1], the diagram of the pressure and speed distribution around the airfoil, which occurs during horizontal flight at zero angle of attack, is justified (Fig. $1)$.

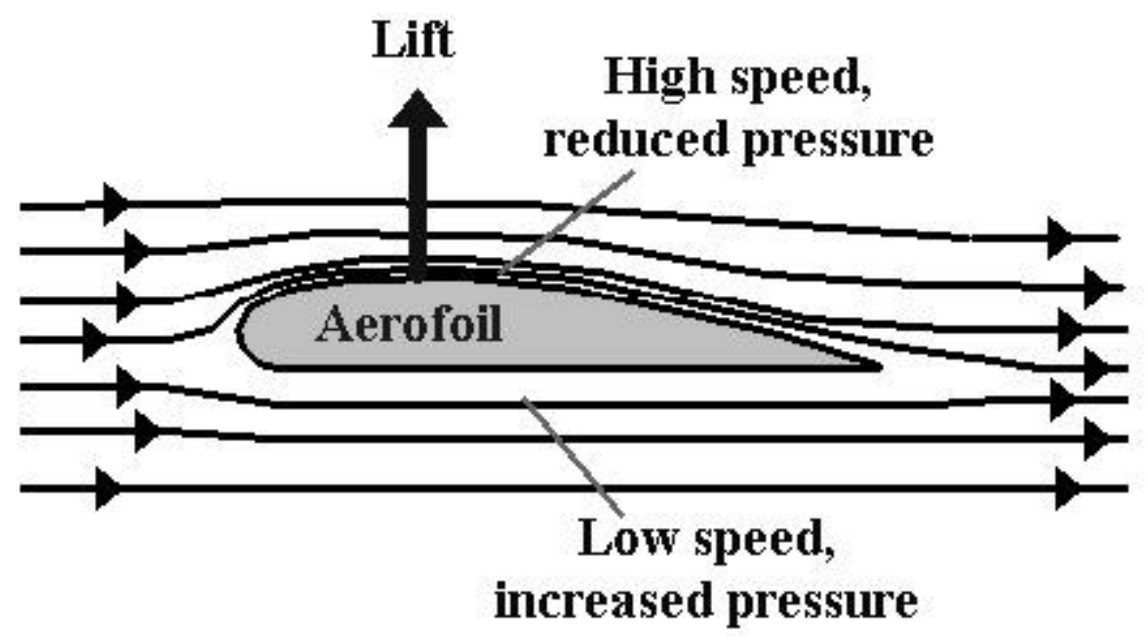

Figure 1. Movement of air around the airplane wing at zero angle of attack. Incorrect distribution of air speed and pressure around the airfoil.

The diagram in figure 1 is well known and it is used in many training manuals to explain the lift of an airplane wing, see for example $[2,3]$. 
The authors of the article [1] tried to explain that the air speed over the airfoil is greater than the air speed under the airfoil. Then, according to the Bernoulli equation, the pressure will be greater under the airfoil and this explains the lift of the wing of the airplane, shown in Fig.1. However, there is no evidence that the air speed is higher over the wing of the airplane. As it turned out, this problem is more than a hundred years old.

If you look at the movement of air around the airfoil in horizontal flight, without paying attention to the inscriptions on Fig. 1, it becomes obvious that the air speed above the wing of the airplane is less than the air speed under the wing of the airplane.

Indeed, figure 1 clearly shows that the wing of the airplane is beating the air from the top of the airfoil, like a hill in an open field. It is obvious that the wind behind the hill is less, therefore, the air speed above the airfoil is less than below the airfoil in horizontal flight. This is how all airplane wings are designed, they have a thickening in front of the wing above the horizon line.

In addition, the air really presses on the airplane wing from above, based on the design of the aerofoil, since the tangent to the aerofoil has an acute angle with the horizon, and from below the air passes parallel to the aerofoil and does not exert additional pressure (Fig. 1). The acute angle between the tangent to the profile and the horizon line at the top of the aerofoil in fact, means that the profile of the airplane wing creates an angle of attack from above for oncoming air flow.

Therefore, the air pressure, based on the diagram, will be higher above the airfoil. Therefore, the "lifting force" shown in figure 1 will be directed downward and will turn into a "falling force". Then the question arises, how to explain the lift of the airplane in horizontal flight and why the airplane does not fall?

The purpose of this article is to describe the lift of an airplane in horizontal flight and answer the question why the airplane remains in the air [1].

\section{The equation of motion of the air and equilibrium of the airplane.}

To solve this problem, we will use the equations of air movement obtained from the minimum action [4], which have the form:

$\rho \frac{\partial v_{j}}{\partial t}=-\frac{\partial P}{\partial x_{j}}-\rho v_{i} \frac{\partial v_{i}}{\partial x_{j}}-\frac{v_{j}}{c^{2}} \frac{\partial P}{\partial t}+\frac{v^{2}}{c^{2}} \frac{\partial P}{\partial x_{j}}-\frac{v_{j} v_{i}}{c^{2}} \frac{\partial P}{\partial x_{i}}$

Here $-\frac{\partial P}{\partial x_{j}}$ is the pressure gradient with a minus sign from Newton's equations;

$f_{j}^{C}=-\rho v_{i} \frac{\partial v_{i}}{\partial x_{j}}-\frac{v_{j}}{c^{2}} \frac{\partial P}{\partial t}-$

centrally symmetric force obtained from the action minimum;

$f_{j}^{V}=\frac{v^{2}}{c^{2}} \frac{\partial P}{\partial x_{j}}-\frac{v_{j} v_{i}}{c^{2}} \frac{\partial P}{\partial x_{i}}-$

vortex force obtained from the minimum action;

$c$ - speed of sound; $v_{j}$ - air speed; $\rho$ - air density; $P$ - pressure. 
In (1), the air viscosity is not taken into account, since it is not essential to explain the lift of the airfoil. Therefore, in the future we will not appeal to the Navier-Stokes equations, but to Euler's equations of hydrodynamics.

As is known [5], the classical Euler equations of hydrodynamics were obtained by differentiating the velocity field as a complex function and have the form:

$$
\begin{aligned}
& \rho \frac{\partial v_{j}}{\partial t}=-\frac{\partial P}{\partial x_{j}}-\rho v_{i} \frac{\partial v_{j}}{\partial x_{i}} \\
& \text { or } \\
& \rho \frac{\partial v_{j}}{\partial t}=-\frac{\partial P}{\partial x_{j}}-\rho v_{i} \frac{\partial v_{i}}{\partial x_{j}}+\rho e_{j i k} v_{i} e_{k m n} \frac{\partial v_{n}}{\partial x_{m}} .
\end{aligned}
$$

It is easy to see that equations (1) and (4') coincide for the case of stationary one-dimensional Bernoulli motion:

$$
-\frac{\partial P}{\partial x_{j}}-\rho v_{i} \frac{\partial v_{i}}{\partial x_{j}}=0
$$

This is where the coincidences between the equations of motion (1) and (4') - end.

As it was shown in [4] and [6], the problem of the general description of turbulence is connected with the incorrectness of Euler's equations. For example, the problem of describing cyclones and anticyclones - the largest vortex movements on Earth is not related to the complexity of solutions to the Euler or Navier-Stokes hydrodynamic equations, as mechanics believe, but to their incorrectness. This was proved in p. 3 [6]. In p. 2 paper [6] show that equations (4) prohibit the very existence of anticyclones, and for cyclones give an incorrect ratio between the kinetic energy of air and pressure when they change, which means that the Euler and Navier-Stokes equations are invalid.

Therefore, in this article, we will not repeat the reasoning of the article [5], but will solve the problem of describing the lift of an airplane in horizontal motion, based on the equations of motion (1) obtained from the minimum action. Especially since this problem is known [1] and obvious (Fig. 1).

The logic of the diagram shown in figure 1 is understandable. The airplane is flying horizontally and does not fall, which means that its wings are affected by the resulting upward force in the form of a pressure difference multiplied by the area of the wings. According to Bernoulli's law, if the air pressure is greater below, under the airfoil, then the air speed must be greater above the airfoil.

However, there is an error in this reasoning. It consists in identifying the air pressure gradient and the force acting on the airfoil from the air. After all, several forces can act on the airfoil from the air, not just the pressure gradient. For example, the wing of an airplane and its fuselage are affected by the Archimedean force directed upwards from the air. It is equal to the density of air multiplied by the volume of the airplane and the acceleration of free fall. The Archimedes force is small and, in the first approximation, it can be ignored, but it is not related to the air pressure gradient that is formed around the airfoil when the airplane moves.

In addition, from figure 1 it is clearly seen that not only is the air speed less above the wing of the airplane, but also the air pressure is greater above the wing of the airplane. Indeed, the oncoming air flow literally presses the airplane down. This is due to the special shape of the airfoil, which has an acute angle between the tangent to the wing profile and the horizon above, 
and below the wing, the air flow passes smoothly, almost without exerting pressure on the airplane. The acute angle between the tangent to the airfoil and the horizon, in fact, means the angle of attack from above, which creates the airfoil. Thus, it is obvious that the air pressure is greater above the wing of the airplane when flying horizontally (fig. 2).

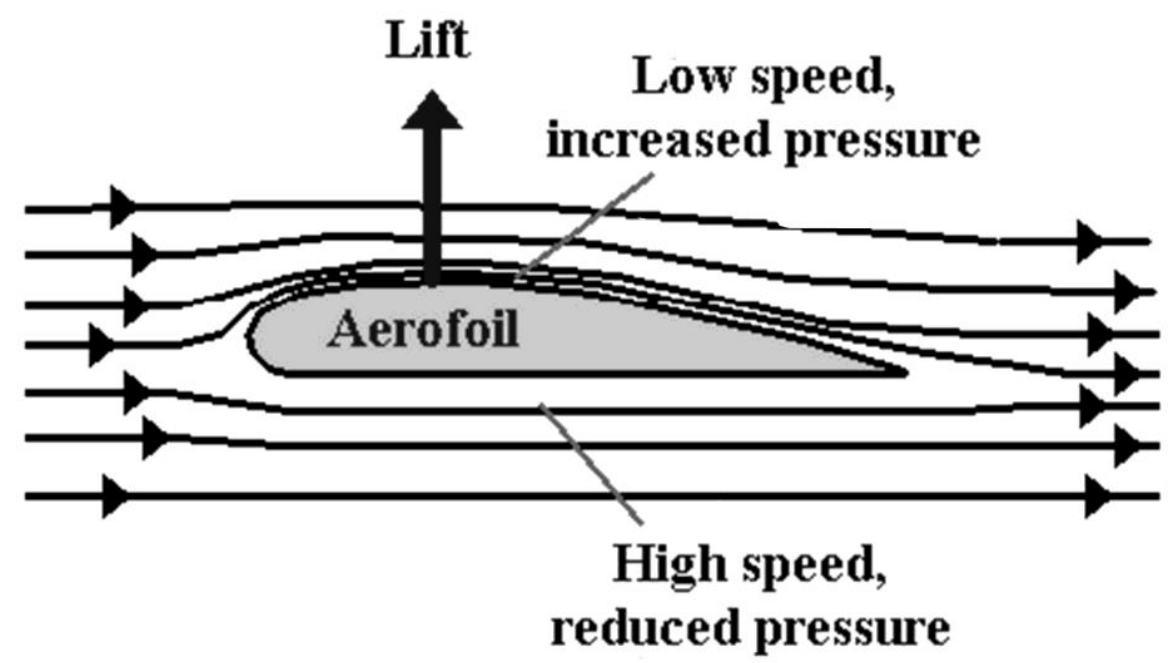

Figure 2. Distribution of air speed and pressure around the airfoil.

Note that we did not use Bernoulli's law in these arguments. The fact that the Bernoulli equations are fulfilled for the air around the airplane during stationary horizontal flight is not obvious, since the air, for example, is strongly compressed and heated when it collides with the airplane. However, in the first approximation, we can assume that the air blowing around the wings of the airplane satisfies the potential movement of Bernoulli (5). Therefore, in the future, for simplicity of reasoning, we will use the expression (5), just as it is used in [1] and [2,3].

In our opinion, the desire to prove the incorrect statement that the air speed is higher above the wing of the airplane (Fig. 1) [1,2] is due to a wrong understanding of equation (5), from which the Bernoulli equation follows. The fact is that during a stationary one-dimensional potential movement of air, the sum of the forces acting on the air is zero (5). Equation (5) follows from equation (1). Thus, according to (5), two forces act on the air: the pressure gradient with a minus sign $-\frac{\partial P}{\partial x_{j}}$, and the potential force $-\rho v_{i} \frac{\partial v_{i}}{\partial x_{j}}$, and this is well known.

However, in the scientific literature on mechanics, the potential force $-\rho v_{i} \frac{\partial v_{i}}{\partial x_{j}}$ is not considered a force. This may be due to the fact that it was obtained from the convective derivative of the velocity field: $v_{i} \frac{\partial v_{j}}{\partial x_{i}}$ [5]. Obviously, it is impossible to obtain the force acting in a continuous medium by differentiating the velocity field. In [6] it was proved that the convective derivative of the velocity field is identically equal to zero. On the other hand, the 
force $-\rho v_{i} \frac{\partial v_{i}}{\partial x_{j}}$ was obtained as a summand of the centrally symmetric force (2) from the action minimum [4]. Therefore, of course $-\rho v_{i} \frac{\partial v_{i}}{\partial x_{j}}-$ this is force.

Potential force $-\rho v_{i} \frac{\partial v_{i}}{\partial x_{j}}$ has the Newton dimension, it stands in the equations of motion (1) and (4') as a force, so of course it is a force.

Why did the aerodynamicists decide that if the sum of all forces acting on a unit volume of air is zero (5), then only the air pressure gradient acts on the wing of the airplane, and the kinetic energy gradient of the air does not act in the form of: $\rho v_{i} \frac{\partial v_{i}}{\partial x_{j}}$ - the question is rhetorical. Most likely, because they wanted to explain the lift force of the airplane (Fig. 1), but how can it be explained if with a potential movement of air that satisfies the Bernoulli equation, the sum of the forces acting on the wing of the airplane from the air is zero (5).

The problem with the diagram shown in Fig. 1 is that it was necessary to explain the lift of the airplane, and there were no other forces other than the pressure gradient. In this case, the air pressure gradient was identified with the air pressure on the wing of the airplane, which is not the same thing. Therefore, the task was to show that the pressure in the air during horizontal flight is greater under the wing of the airplane [1-3], although it is obvious to everyone that this is not the case (Fig. 2).

The potential force $-\rho v_{i} \frac{\partial v_{i}}{\partial x_{j}}$ was not considered a force, but was considered a certain "ploy" associated with the replacement of Lagrange coordinates with Euler coordinates. As shown in [6] this is not true. The potential force exists and must be taken into account when moving a continuous medium. It is responsible for the flat rotation of air in the form of cyclones and anticyclones when the pressure in the atmosphere changes [6].

If we take into account the potential force, then for a potential stationary air flow it does not matter where the pressure is greater below the airfoil or above the airfoil. Since according to the Bernoulli equations, which are referred to in [1] and [2,3], the sum of the forces acting on the wing of the airplane from the air at its potential flow is zero (5).

Thus, to describe the lifting force of the airplane, we have only one vortex force: $f_{j}^{V}=\frac{v^{2}}{c^{2}} \frac{\partial P}{\partial x_{j}}-\frac{v_{j} v_{i}}{c^{2}} \frac{\partial P}{\partial x_{i}}(1,3)$, since the Archimedean force, equal to the weight of the displaced air, is small, and it is usually not taken into account $[2,3]$. 
Note that the second term of the vortex force: $-\frac{v_{j} v_{i}}{c^{2}} \frac{\partial P}{\partial x_{i}}$ (3) is zero when the airplane is moving horizontally, since the vector $\frac{\partial P}{\partial x_{i}}$ is directed vertically, and the air velocity $v_{i}$ is directed horizontally, near the airfoil. Therefore, the scalar multiplication $v_{i} \frac{\partial P}{\partial x_{i}}$ is zero.

The first term of the vortex force (3): $\frac{v^{2}}{c^{2}} \frac{\partial P}{\partial x_{j}}-$ is not equal to zero. This force keeps the airplane in horizontal flight at high speed.

Indeed, note that the force $\frac{v^{2}}{c^{2}} \frac{\partial P}{\partial x_{j}}$ is directed upwards, since the air pressure is higher above the airfoil. To get the force acting on the airfoil, it is necessary to sum up the action of the volumetric force $\frac{v^{2}}{c^{2}} \frac{\partial P}{\partial x_{j}}$ from the air above and below the wing of the airplane.

Integrating the expression $\frac{v^{2}}{c^{2}} \frac{\partial P}{\partial x_{j}}$ along the vertical axis, we assume that the speed value does not change much during integration then in the first approximation we get the force acting on the unit area of the airfoil in the form $\frac{v^{2}}{c^{2}}\left(P-P_{0}\right)$. Where $P_{0}$ - is the pressure under the wing of the airplane and $P$ - is the pressure over the wing of the airplane. The pressure $P_{0}$ coincides with the air pressure at infinity $P_{\infty}$ since under the wing of the airplane, the pressure practically does not change during horizontal flight. The pressure $P$ during horizontal flight is higher above the wing of the airplane, this follows from the design of the airfoil (Fig. 1).

Now we need to multiply the pressure from the air on the wing of the airplane $\frac{v^{2}}{c^{2}}\left(P-P_{0}\right)$ by the area of the wings $2 S$ and we get the lift of the airplane in the form $2 S \frac{v^{2}}{c^{2}}\left(P-P_{0}\right)$ when moving horizontally in a straight line at a speed of $v$. Above the wing, the pressure is higher, so this force is directed upwards, which was necessary to prove.

Thus, the sum of the forces acting on the airplane in the projection on the vertical axis, in the first approximation, without the Archimedean force, has the form

$$
-M g+2 S \frac{v^{2}}{c^{2}}\left(P-P_{0}\right)=0 \text {. }
$$

Now it becomes clear why you need to thicken the top of the wing of the airplane (Fig. 2), so that the pressure is higher above, and not below the wing of the airplane. 
When constructing an airfoil, developers achieve potential airflow around the wing of the airplane without vortex movement around the wing of the airplane. Then the vortex force $\frac{v^{2}}{c^{2}} \frac{\partial P}{\partial x_{j}}$ compensates for the force of gravity and keeps the airplane in the air at high speeds.

\section{The turbulent trace of the plane.}

The problem of describing the lift force of an airplane wing is directly related to the problem of describing the vortex trail of an airplane.

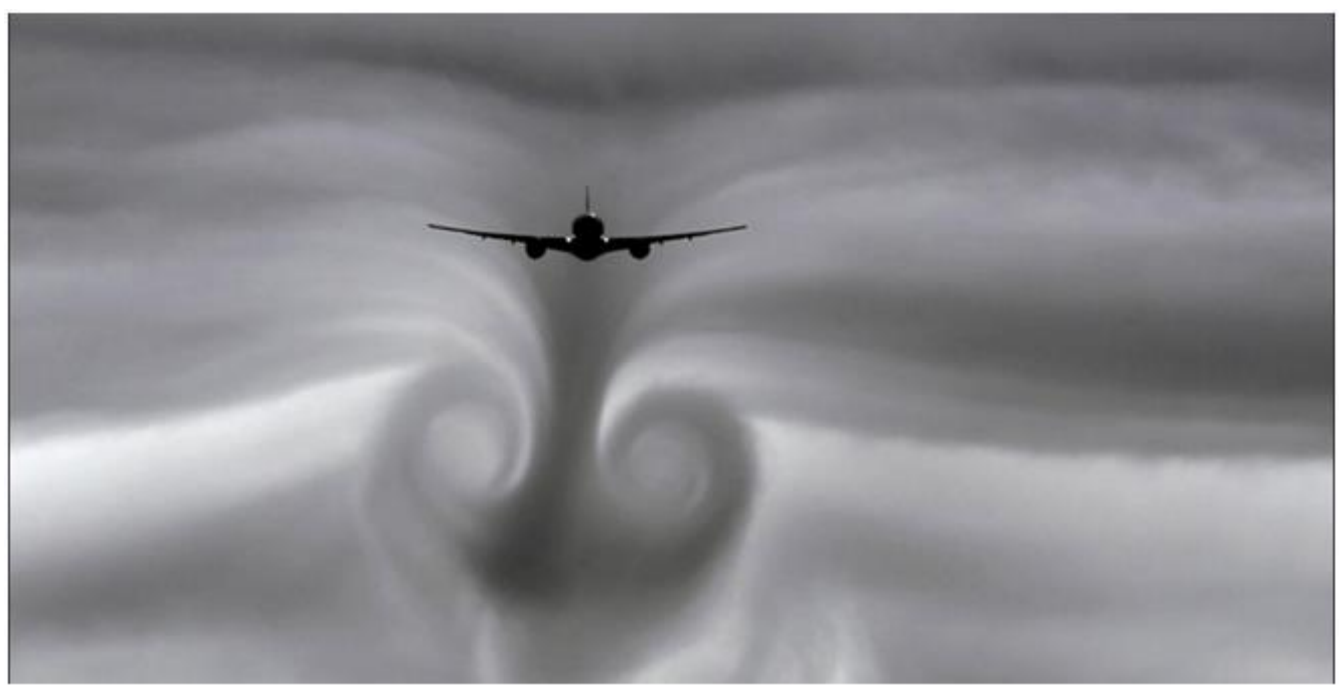

Figure 3. In this photograph, the vortex air movement is clearly visible in the form of two vortices with horizontal axes that follow the two wings of the airplane.

When the plane flies, a vortex movement of air forms behind its wings (Fig. 3), Q.E.D. Indeed, it is the wings that prevent the occurrence of vortex air movement as a result of the action of vortex force (3).

While the plane is flying, the vortex force component $\frac{v^{2}}{c^{2}} \frac{\partial P}{\partial x_{j}}$ compensates for the airplane's gravity and does not act on the air. It's like hiding under the wing of an airplane. As soon as the airplane has passed, the vortex force (3) acts on the air, it accelerates it, and together with the central force (2) makes air to move in the form of vortices, as shown in (Fig. 3).

Note that the Euler's equations (4) and, consequently, the Navier-Stokes equations cannot describe vortex motion (Fig.3). The invalidity of the Navier-Stokes equations is reflected in the Millennium problem: http://www.claymath.org/millenniumproblems/navier\%E2\%80\%93stokes-equation. Let's prove this statement.

Let an airplane fly in the direction of the axis $O X$, and the pressure gradient acts on the air behind the airplane vertically in the direction of the axis $O Z$. Then the forces acting on the air behind the airplane, according to equations (4), lie in the plane $X Z$. In this case, there is no force in the direction of the axis $O Y$ - in the horizontal plane perpendicular to the fuselage of the airplane. This is easy to validate. 
Firstly, the pressure gradient $\frac{\partial P}{\partial x_{j}}=\frac{\partial P}{\partial x_{3}}$ is directed vertically, hence there is no force in the horizontal plane due to the pressure gradient

Secondly, the convective derivative of the velocity field $v_{i} \frac{\partial v_{j}}{\partial x_{i}}$ in the direction of the axis $O Y$ is zero. Indeed, since the component of the velocity field $v_{2}$ in the $O Y$ direction is zero, the convective derivative of the velocity field $v_{i} \frac{\partial v_{2}}{\partial x_{i}}$ will also be zero. Hence, Euler's equations (4) cannot describe the trace of an airplane (Fig. 3) since in classical hydrodynamics there is no force imparting centripetal acceleration to air in the direction of the axis $O Y$, which swirls the vortex.

Obviously, the viscosity accounting in the Navier-Stokes equations is not able to correct this situation, since the viscosity in the air is very small. In addition, due to the isotropy of the air, the viscosity can't explain the resulting anisotropic pattern of the airplane trace (Fig. 3). The invalidity of the Euler and Navier-Stokes equations is that they can't describe the vortex trace of an airplane (Fig.3), as well as plane vortex movements [6].

On the other hand, the potential force $-\rho v_{i} \frac{\partial v_{i}}{\partial x_{j}}(1,2)$, which can be written as $-\frac{\rho}{2} \frac{\partial v^{2}}{\partial x_{j}}$, easily explains the presence of a centripetal force in the air that occurs behind the airplane in the direction $O Y$.

Indeed, as a result of the vertical force $\frac{v^{2}}{c^{2}} \frac{\partial P}{\partial x_{3}}$ caused by the pressure gradient, there is a vertical flow of air behind the wing of an airplane. At the same time, vertical flow does not occur away from the airplane on the axis $O Y$, at a distance greater than the wing of the airplane therefore, a gradient of the speed square $\frac{\partial v^{2}}{\partial x_{2}}$ along the axis $O Y$ is formed. Consequently, there is a force $-\frac{\rho}{2} \frac{\partial v^{2}}{\partial x_{2}}$ directed along the axis $O Y$ from the fuselage of the airplane. This force $-\frac{\rho}{2} \frac{\partial v^{2}}{\partial x_{2}}$ together with the vortex force (3) swirls the air into the vortices shown in (Fig. 3).

Note that the potential force $-\rho v_{i} \frac{\partial v_{i}}{\partial x_{j}}(1,2)$ obtained from the action minimum [4] and the convective derivative of the velocity field multiplied by the density $-\rho v_{i} \frac{\partial v_{j}}{\partial x_{i}}$ in Euler's 
equations (4) are two different forces. The force $-\rho v_{i} \frac{\partial v_{j}}{\partial x_{i}}$ acts on the air along its movement, and the potential force $-\rho v_{i} \frac{\partial v_{i}}{\partial x_{j}}$ acts on the air perpendicular to its movement, which explains the vortex trail of the airplane (Fig. 3). As it was shown in [5], this is and the problem of describing plane vortex movements of air in the atmosphere.

However, as shown in this paper, difference in descriptions of continuous medium movement in (1) and (4) was deeper and it is related to relativism. Therefore we will devote the rest of this article to discussing relativism and the vortex force that keeps an airplane in the air.

\section{Lifting force of the airplane in horizontal flight.}

We will arrange, as they say in such cases, a debriefing. As a result of taking into account the vortex force (3) in the air, the lift force acting on the wings of the airplane (6) was obtained: $2 S \frac{v^{2}}{c^{2}}\left(P-P_{0}\right)$. It differs from the lift force is written in the textbooks on aerodynamics: $2 S\left(P_{0}-P\right)[2,3]$.

Firstly, these two forces differ in direction, the force $2 S \frac{v^{2}}{c^{2}}\left(P-P_{0}\right)$ is directed up and the force $2 S\left(P_{0}-P\right)$ is directed down. Secondly, these forces differ by a relative multiplier $v^{2} / c^{2}$.

Let's examine the extreme cases for lift in (6), depending on the speed of the airplane. Let's assume that the speed of an airplane is ten times less than the speed of sound, i.e. less than $30 \mathrm{~m} / \mathrm{c}$. Then the relative coefficient $v^{2} / \mathrm{c}^{2}$ will be less 0.01 , since the speed of sound is of the order of $300 \mathrm{~m} / \mathrm{c}$. In this case, the lift is very small and the airplane will not even be able to flay up off at this speed (meaning a large plane).

Let's now consider another extreme case, when a large airliner flies horizontally at a speed close to the speed of sound. Then in the first approximation, you can put $v=c$. In this case, the pressure gradient in the equations of motion (1) is reduced, since $-\frac{\partial P}{\partial x_{j}}+\frac{v^{2}}{c^{2}} \frac{\partial P}{\partial x_{j}}=0$ at $v=c$, and the airplane is held in the air only by the potential force given by the velocity gradient: $-\rho v_{i} \frac{\partial v_{i}}{\partial x_{j}}(1)$

Note that this result does not depend on the assumption that the movement of air around the plane satisfies the Bernoulli equation. When the speed of the airplane is nearby to the speed of sound, the pressure gradient is annulated in the equations of motion for air (1) when the plane is moving horizontally. Therefore, the wings of all airplanes are arranged so that when flying horizontally, the air speed is greater under the wing of an airplane (Fig. 2), otherwise, the airplane simply can not be in the air when flying horizontally. 
In this paper, we used the Bernoulli equation for methodological reasons to clearly explain the action of the vortex force: $\frac{v^{2}}{c^{2}} \frac{\partial P}{\partial x_{j}}$. It is obvious that in general, it is necessary to set boundary conditions and solve the problem of equilibrium for the airplane and for the air when the airplane is flying horizontally.

However, schematically, in the first approximation, we can assume that the air near the wing of the airplane moves potentially, without vortices, and the condition (5) is fulfilled. Then in the equilibrium equation for an airplane along a vertical axis, which contains three forces related to the movement of air $-\frac{\partial P}{\partial x_{j}}-\rho v_{i} \frac{\partial v_{i}}{\partial x_{j}}+\frac{v^{2}}{c^{2}} \frac{\partial P}{\partial x_{j}}$ will remain one of the vortex strength. This equation is easily solved as shown above.

The fact that the vortex force (3) is the lifting force was known from [4], since it is responsible for the lifting force in tornadoes.

In tornadoes, the lifting force is equal to $\frac{v_{\perp}^{2}}{c^{2}} \frac{\partial P}{\partial x_{3}}$ where $v_{\perp}$ is the horizontal vortex velocity in the tornado, and the pressure gradient $\frac{\partial P}{\partial x_{3}}$ is directed along the vertical axis upwards towards the expansion of the funnel of the tornado. At the same time, in [4] it was assumed just as for an airplane, that the gradient of air pressure along the vertical axis is compensated by the gradient of the kinetic energy of air rotation in the tornado, since the tornado, in the first approximation, can be considered a stable vortex movement of air.

Thus, the lift in a tornado is the same as the lift in an airplane. The only difference is that in a tornado the air moves in a circle in a horizontal plane, and the airplane flies straight and itself creates forces acting on it from the air. Therefore, it was not at all obvious that the lifting force for an airplane could be a vortex force (3). This problem was solved thanks to the statement of the question in the article [1].

On the other hand, everything is less obvious in a tornado than in a situation with an airplane, since it is difficult to check something in a tornado. This is the main problem associated with studying tornadoes, since the tornado breaks all the instruments before they have time to measure anything.

There is a scale of intensity of tornadoes it is called The Fujita scale [7]. The Fujita scale clearly shows that the speed at which a large passenger plane fly up $270 \mathrm{~km} / \mathrm{h}$ and the wind speed in a tornado F3, at which it is able to lift large cars $254 \mathrm{~km} / \mathrm{h}$, are of the same order. From this we can conclude that the lifting force in tornadoes is of the same nature as the lifting force of an airplane.

It is obvious that the lift effect in (6) is related to the relative multiplier $v^{2} / c^{2}$ before the pressure gradient in the first term of the vortex force $\frac{v^{2}}{c^{2}} \frac{\partial P}{\partial x_{j}}$ (3). Of course, you can get more lift by increasing the pressure gradient but if the speed is small then the pressure gradient alone is 
not enough. Therefore, both factors are important here: the pressure gradient $\frac{\partial P}{\partial x_{j}}$ which is related to the airfoil structure, and the relative coefficient $v^{2} / c^{2}$ which is related to the speed of the airplane.

Note that the vortex force (3) cannot be obtained using mechanistic representations of a continuous medium as a "set of molecules". Part of the vortex force $\frac{v^{2}}{c^{2}} \frac{\partial P}{\partial x_{j}}$ is directed from low pressure to high pressure, so mechanical representations do not work here.

\section{Explanation and coclusions.}

In conclusion, let us recall how the forces (2) and (3) acting in a continuous medium appeared and why they are relativistic - they have a relative multiplier $v^{2} / c^{2}$. First, we constructed a minimal interaction [8] induced by a subgroup of translations in the form of an expanded derivative, in accordance with the Pauli principle [9]. It is known that the electrodynamics described by Pauli is a relativistic theory [10]. After constructing the minimal interaction induced by the translation subgroup [7], the forces of this interaction were calculated from the action minimum in the same way as the Coulomb and Lorentz forces are calculated in the field theory [9].

However, the first relativism in this theory [4, 8] was introduced by Kadic-Edelen [11]. By analogy with electrodynamics they wrote down the pseudo-Lorentz calibration condition, with the only difference that $c$ - here is the speed of sound, not the speed of light. The pseudoLorentz calibration condition is physical, for example, it follows that mechanical waves in a continuous medium propagate at the speed of sound [4].

Therefore, relativism is initially present in the expression for forces derived from the action minimum: $-\rho v_{i} \frac{\partial v_{i}}{\partial x_{j}}-\frac{v_{j}}{c^{2}} \frac{\partial P}{\partial t}+\frac{v^{2}}{c^{2}} \frac{\partial P}{\partial x_{j}}-\frac{v_{j} v_{i}}{c^{2}} \frac{\partial P}{\partial x_{i}}$ (1) [4]. Note that the first term is the potential force which had been known since the time of Bernoulli and Euler, and the other terms are relativistic forces, all of which contain the speed of sound squared in the denominator.

At high speeds comparable to the speed of the sound at which large airplane is fly, the vortex force component $\frac{v^{2}}{c^{2}} \frac{\partial P}{\partial x_{j}}$ (3) is not small. It is the lifting force for the airplane in horizontal flight. To increase lift, you need to increase the speed of the airplane or increase the air pressure gradient. Given that in the first approximation, it can be assumed that the air moves potentially in the area of the wing of the airplane (5), an increase in the pressure gradient in the air can be achieved by increasing the air velocity gradient.

Thus, it is necessary to make such a design of the airfoil that the speed above the wing is less than below the wing of the airplane (Fig. 2). Our airplane designers successfully coped with this task, without theoretical aerodynamics [1-3]. Now that there is an explanation for such a wing design in an airplane, it will be possible to design new airplane models using computer calculations, without resorting to expensive and not always possible experiments using wind tunnels. 


\section{References}

1. E. Regis, No One Can Explain Why Planes Stay in the Air Scientific American (February 2020). https://www.scientificamerican.com/article/no-one-can-explain-whyplanes-stay-in-the-air/

2. C.E. Dole, J.E. Lewis, J.R. Badick, B.A. Johnson, Flight Theory and Aerodynamics (Wiley 2017).

3. J.D. Anderson, Jr, Fundamentals of Aerodynamics (McGraw-Hill, 2011).

4. A.Y. Braginsky, The vortex motion of the continuous medium, depending on the pressure change. PTEP 2020 (6), 063J03 (2020). DOI: 10.1093/ptep/ptaa076

https://www.researchgate.net/publication/332253999

5. L.D. Landau, E.M. Lifshitz, Hydrodynamics, Volume 6. (Nauka Moscow, 1988).

6. A.Y. Braginsky, Plane vortex motion of a continuous medium. Description of air rotation in cyclones and anticyclones. ZAMP 70, 177 (2019).

7. T.W. Schmidlin Fujita Tornado Scale. In: Bobrowsky P.T. (eds) Encyclopedia of Natural Hazards. Encyclopedia of Earth Sciences Series. Springer, Dordrecht (2013).

8. A.Y. Braginsky, Electron pairing in a Cooper pair as attraction of oppositely directed quantum momenta. Moscow University Physics Bulletin 74 (6), 566 (2019).

9. W. Pauli, Relativistic field theories of elementary particles. Revs. Mod. Phys. 13, 203 (1941).

10. L.D. Landau, E.M. Lifshitz, Field Theory, Volume 2. (Nauka Moscow, 1987).

11. A. Kadić, D.G.B. Edelen, A Gauge Theory of Dislocations and Disclinations. Lecture Notes in Physics. Heidelberg. Springer 174, 168 (1983).

\section{Declarations}

- Availability of data and materials

The datasets generated and/or analysed during the current study are available in the https://www.researchgate.net/profile/Alexander_Braginsky

- Competing interests

The authors declare that they have no competing interests

- $\quad$ Funding

No funding

- Authors' contributions

Only one author

- Acknowledgements 
Not applicable

- Authors' information (optional) 
Figures

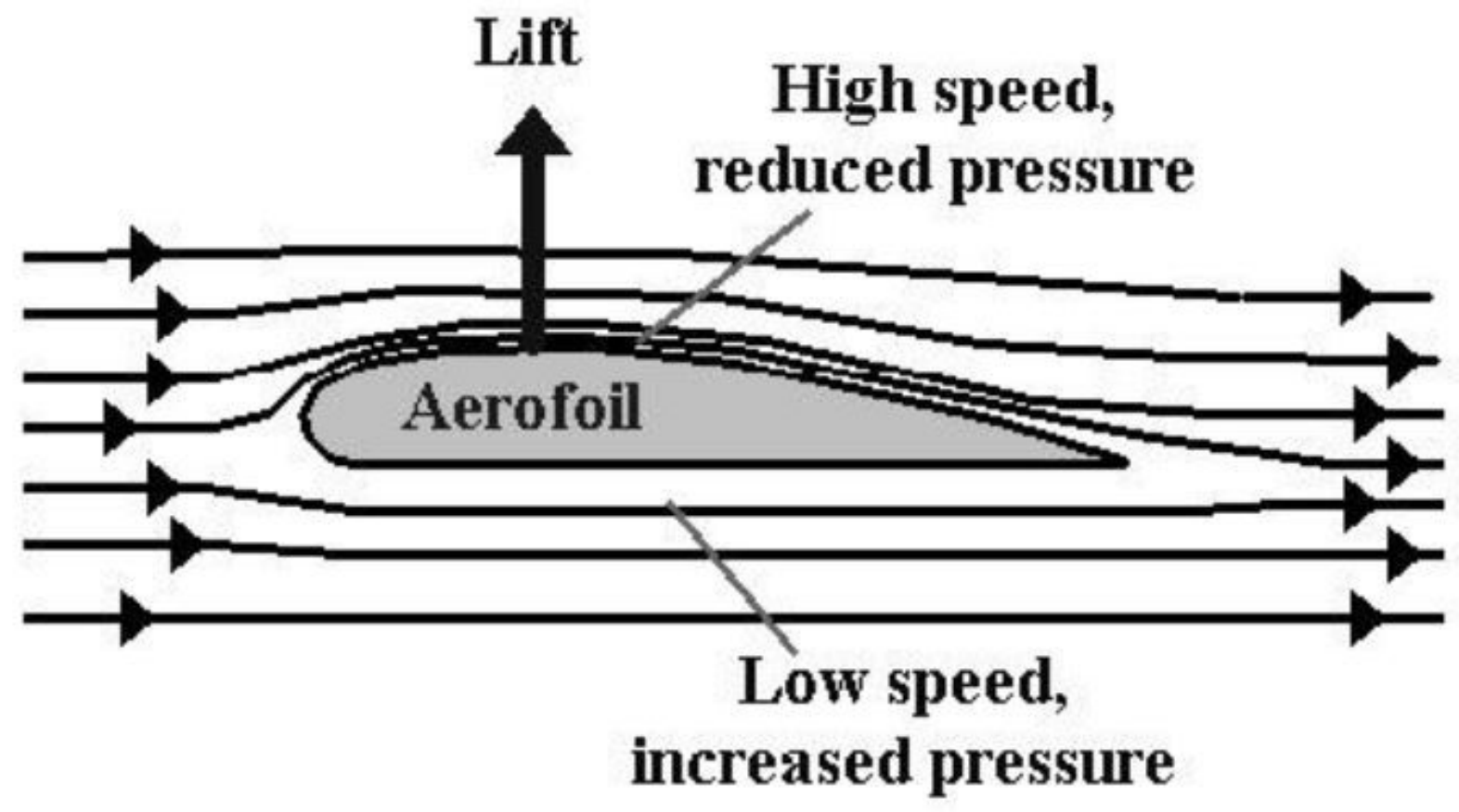

Figure 1

Movement of air around the airplane wing at zero angle of attack. Incorrect distribution of air speed and pressure around the airfoil. 


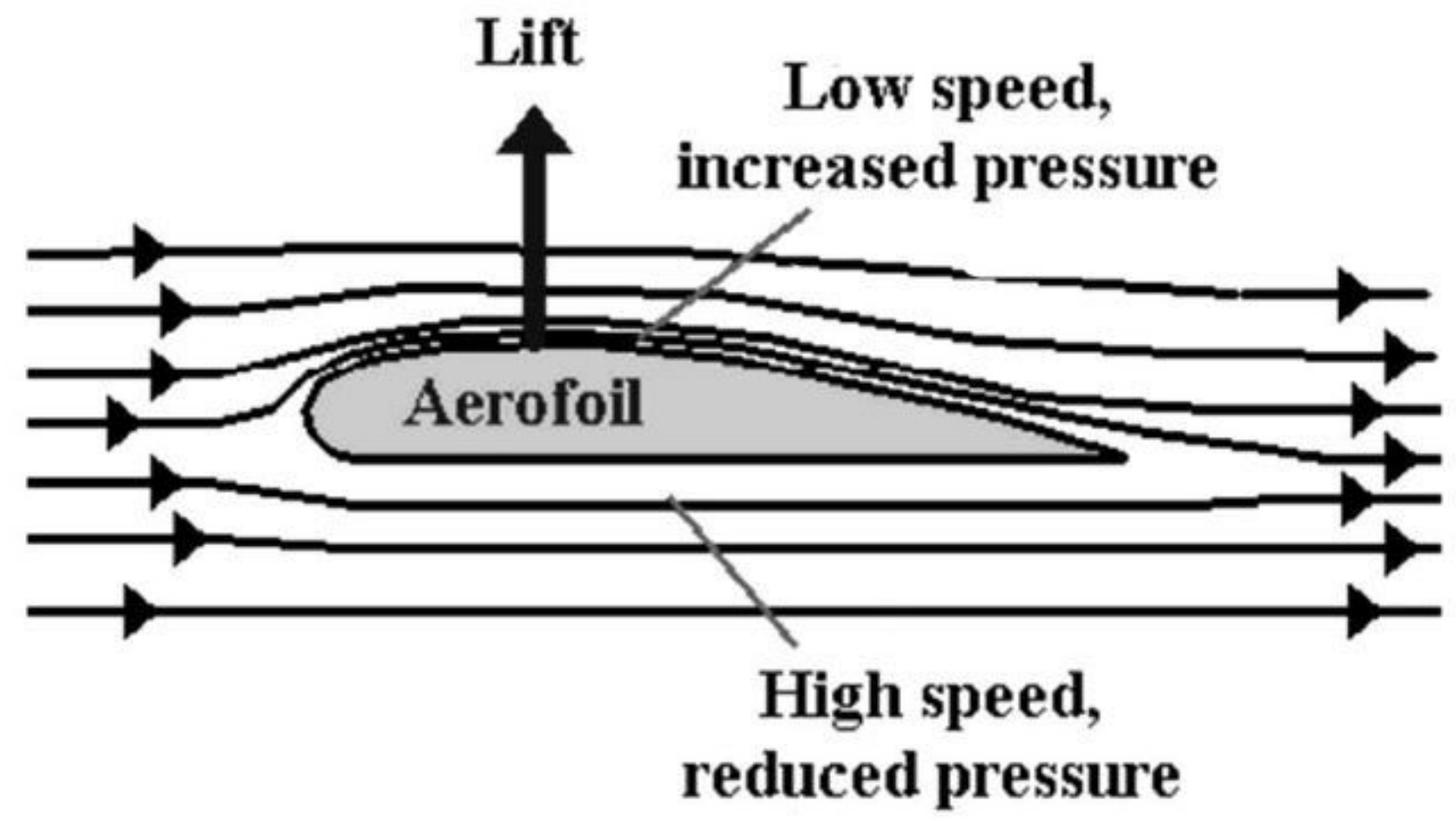

Figure 2

Distribution of air speed and pressure around the airfoil.

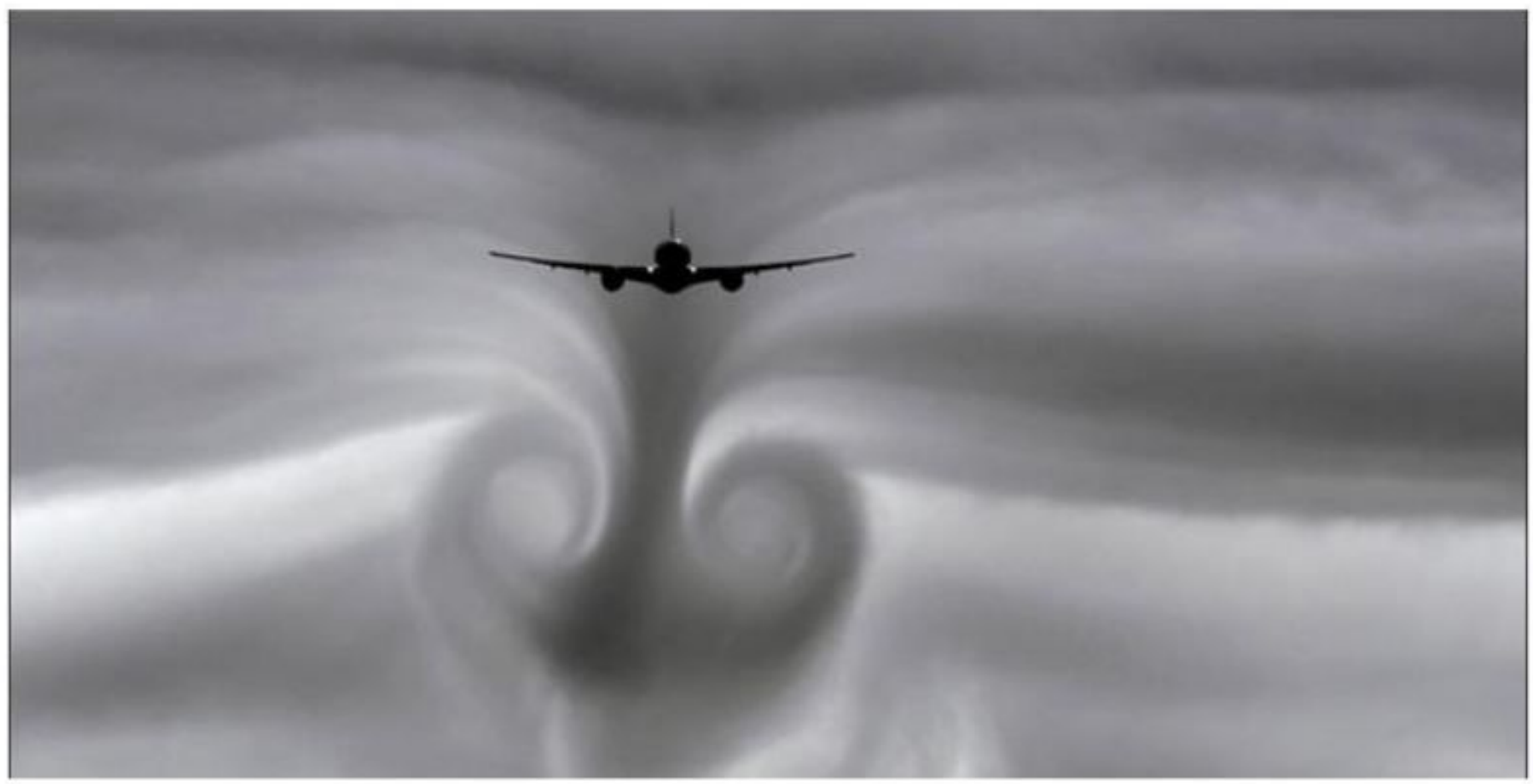

Figure 3 
In this photograph, the vortex air movement is clearly visible in the form of two vortices with horizontal axes that follow the two wings of the airplane. 